\title{
A PRELIMINARY EXAMINATION OF AGE-RELATED DIFFERENCES IN PERCEIVED COMPLEXITY AT FATAL-CRASH INTERSECTIONS
}

\author{
Timothy J. Wright ${ }^{1}$, William J. Horrey ${ }^{2}$, \& David A. Lombardi ${ }^{2}$ \\ ${ }^{1}$ Dunlap and Associates, Inc. \\ Stamford, CT, USA \\ ${ }^{2}$ Liberty Mutual Research Institute for Safety \\ Hopkinton, MA, USA \\ Email: timwright167@gmail.com
}

\begin{abstract}
Summary: Younger and older drivers are both overrepresented in fatal crashes that occur at intersections, however, after adjusting for other significant factors (i.e., being at fault type of road, weather, lighting) the increased risk cannot be fully accounted for older drivers, nor does frailty. Thus, increased risk for older drivers could be due to their agerelated cognitive declines and possible differences in perceptions of intersections. The current study examines whether older drivers' perceived complexity of intersections differed quantitatively and qualitatively from younger drivers' perceived complexity of the same intersections. Coordinates of a random sample of intersections where at least one fatality occurred over a three-year period from the US Fatality Analysis Reporting System (FARS) were identified and Google Earth was used to extract still images of each intersection. The complexity of these intersection images were rated by a sample $(\mathrm{N}=38)$ of younger (age 18-35) and older drivers (age 65+). Inter-rater reliability for each group was calculated. In addition, individual intersection images with the largest and smallest age differences were qualitatively examined. Results suggest that older drivers view the complexity of intersections differently than younger drivers. Overall, older drivers were less reliable and scored nominally higher on average in their complexity ratings than younger drivers. Moreover, older drivers tended to rate rural or residential intersections as being more complex than younger drivers; whereas younger drivers tended to rate urban intersections as being more complex. Future work should account for these age differences in perceived intersection complexity.
\end{abstract}

\section{INTRODUCTION}

Older adults are overrepresented in fatal crashes at intersections. By the age of 85 , fatal intersection crash risk is almost doubled after controlling for significant factors including time of day, day of week, type of road, type of trafficway, road alignment, weather conditions, lighting conditions, or being deemed at fault for the crash; whereas for younger drivers, intersection crash risk is not significantly increased after controlling for these factors (Lombardi et al., 2017). Moreover, increased fragility of older drivers does not appear to fully explain their increased fatal crash risk (Li et al., 2003), however perceptual and cognitive declines that occur with age (Salthouse, 2004) may be likely contributors. Consistent with this hypothesis, the cognitive abilities of older adults have been linked to increased crash risk (Owsley et al., 1998). Concomitant with these declines, it is also possible that older drivers perceive intersections and their relative complexity differently from younger drivers.

The current study is a preliminary examination of the degree to which perceived intersection complexity differs quantitatively and qualitatively among younger and older drivers. A random 
sample of geocoded intersections where at least one fatality occurred in the US between the years 2011 and 2013 were used for the ratings. Overhead zoomed images of these intersections from Google Earth were extracted and a sample of older and younger drivers were asked to rate the complexity of these intersections based on a scale. We hypothesize that if older drivers perceive intersections differently from younger drivers, then these two age groups should differ in mean intersection complexity rating scores and the reliability for older drivers should be lower (i.e., complexity scores should be more divergent across older raters). Moreover, if the age groups perceive intersections differently, there should be clear qualitative differences in the way the drivers in these groups determine a complexity rating.

\section{METHOD}

\section{Participants}

Forty licensed drivers (20 female/20 male) were recruited to complete a 3-hour laboratory study and were compensated for their participation with a $\$ 60$ gift card. Half were younger adults $(M$ age $=25$ years, $S D=6$, Range $=18-35)$, and the other half were older adults ( $M$ age $=69$ years, $S D=2$, Range $=65-72)$. Gender was balanced in each age group. Data for one older adult was later excluded due to failing to complete the study. In addition, another older driver's data was excluded due to a technical issue in data collection. All participants reported normal or corrected-to-normal vision and driving at least 30 miles per week (younger adults: $M=186$ miles, $S D=149$; older adults: $M=143$ miles, $S D=107$ ).

\section{Stimuli}

From among all fatal intersection crashes during the period of 2011-2013 in the FARS database, we randomly selected two crash intersections from each state and Washington, DC (a total of 102 intersections; one image was deemed unusable). To capture (screen-shot) available images of these locations, the lateral and longitudinal GPS coordinates of each intersection were entered into Google Earth. Multiple perspectives of most intersections were captured (North, East, South, West, and overhead); however, only the overhead ("bird's-eye") view is examined in the current analysis.

\section{Procedure}

Upon arrival at the laboratory, participants (up to six individuals in a single session) were briefed regarding the general purpose of the study. After providing informed consent, they completed demographic questions before starting the intersection rating task (both administered via ePrime software version 2.0; Psychology Software Tools, Inc., Sharpsburg, PA). For the rating task, participants were instructed to use their judgment and provide a complexity rating on a scale of 1 (least complex) to 10 (most complex) for each intersection image. Ten equidistant response boxes (containing the numbers 1-10) were presented above each intersection image for participants to provide their complexity ratings with a mouse-click while looking at the current image. Participants were further instructed to consider and make assumptions regarding the following factors in their ratings (even if in some cases they could not be observed from the image presented): 
1) traffic environment (urban vs. rural setting),

2) angle of intersecting roads,

3) road curvature near or within intersections,

4) number of lanes of travel,

5) presence of staggered junction (e.g., road does not align on opposing sides of intersection),

6) lane continuity violations (e.g., multiple lanes merge together on opposite side of intersection),

7) presence of dedicated turn lanes (e.g., lanes with arrows painted in them),

8) presence and nature of traffic control devices (e.g., traffic lights, signs),

9) visibility obstruction (e.g., buildings, hills, environmental features).

Before starting the rating process for the experimental images, participants were given an opportunity to practice rating the complexity of 8 unique practice intersection images. Following practice, participants were given a final opportunity to ask the experimenter questions before beginning the experimental blocks ( 5 total). After each block, participants were provided with the opportunity for a break.

\section{Inter-rater Reliability Calculation}

To examine the extent that each of the thirty-eight licensed drivers were consistent with the other drivers in their ratings of perceived complexity, the percent agreement coefficient as a measure of Intraclass-Correlation (ICC) was calculated. To calculate this measure, the subjective complexity ratings (ranging from 1-10) of younger and older drivers were categorized as either low (1-3), medium (4-7), or high (8-10). These classifications were based on a pilot study involving eleven independent raters. Once these classifications were assigned, the percent agreement coefficient (overall and by age group) was calculated between raters, which takes into consideration the within and between rater variance (Fleiss, 1981).

\section{RESULTS}

\section{Quantitative Age Differences}

Table 1 below presents descriptive and inter-rater reliability statistics for each age group. Overall older drivers scored intersections nominally higher and were much less reliable in their \% agreement in perceived complexity of the intersections than younger drivers. This suggests that older drivers are likely viewing the intersections qualitatively differently from their younger counterparts.

\section{Qualitative Age Differences}

To understand the qualitative differences in these two age groups in their perception of intersection complexity, individual intersections with the largest age group differences (e.g., older adults rating the intersection more complex than younger adults or vice versa) were compared to those with the smallest or no age differences. 
Table 1. Descriptive and inter-rater reliability statistics

\begin{tabular}{lcc}
\hline & & \\
Age Group & Complexity Rating Mean (SD) & \% agreement \\
\hline Younger Drivers & $4.40(1.20)$ & .54 \\
Older Drivers & $4.81(0.75)$ & .36 \\
OVERALL & $4.60(1.02)$ & .47 \\
\hline
\end{tabular}

Figure 1 below presents images and mean complexity ratings for these intersections for each age group. Examining Figure 1, it becomes clear that the intersections with the largest age differences tended to be rural or residential roadways with few lanes, low traffic, and little roadside infrastructure. On the contrary, the intersections with the smallest age differences or ones in which younger adults rated the intersections more complex than older adults were urban or highway roadways, typically with more lanes, more traffic, and more infrastructure. In sum, clear qualitative differences distinguish what older adults view as a complex intersection compared to what younger adults view as a complex intersection.

\section{DISCUSSION}

The current study examines a potential explanation for age differences in intersection crash risk (Lombardi et al., 2017) by showing that older drivers may perceive the complexity of intersections differently than younger drivers. Importantly, results of this study suggest that older adults may underestimate the complexity of more difficult intersections. This underestimation could potentially lead an older driver to overestimate their ability to accommodate the relative demands (e.g., Fuller, 2005), making it more likely that they will choose to navigate through these complex intersections.

Why are older adults choosing counter-intuitive criteria when formulating their complexity ratings? It is possible that older adults were effectively following directions and projecting the difficulty of intersections in a different context than what was physically pictured. For example, while these pictures are in the daytime and visibility is not an issue in the situation pictured, visibility could become an issue at night for the intersections older adults rated as more complex. In the rural or residential intersections, street lights are not as common as in the urban or highway intersections. This potential strategy of older drivers is consistent with the declines in visual acuity and contrast sensitivity that occur with age. Moreover, the potential strategy is 


\section{Older > Younger}

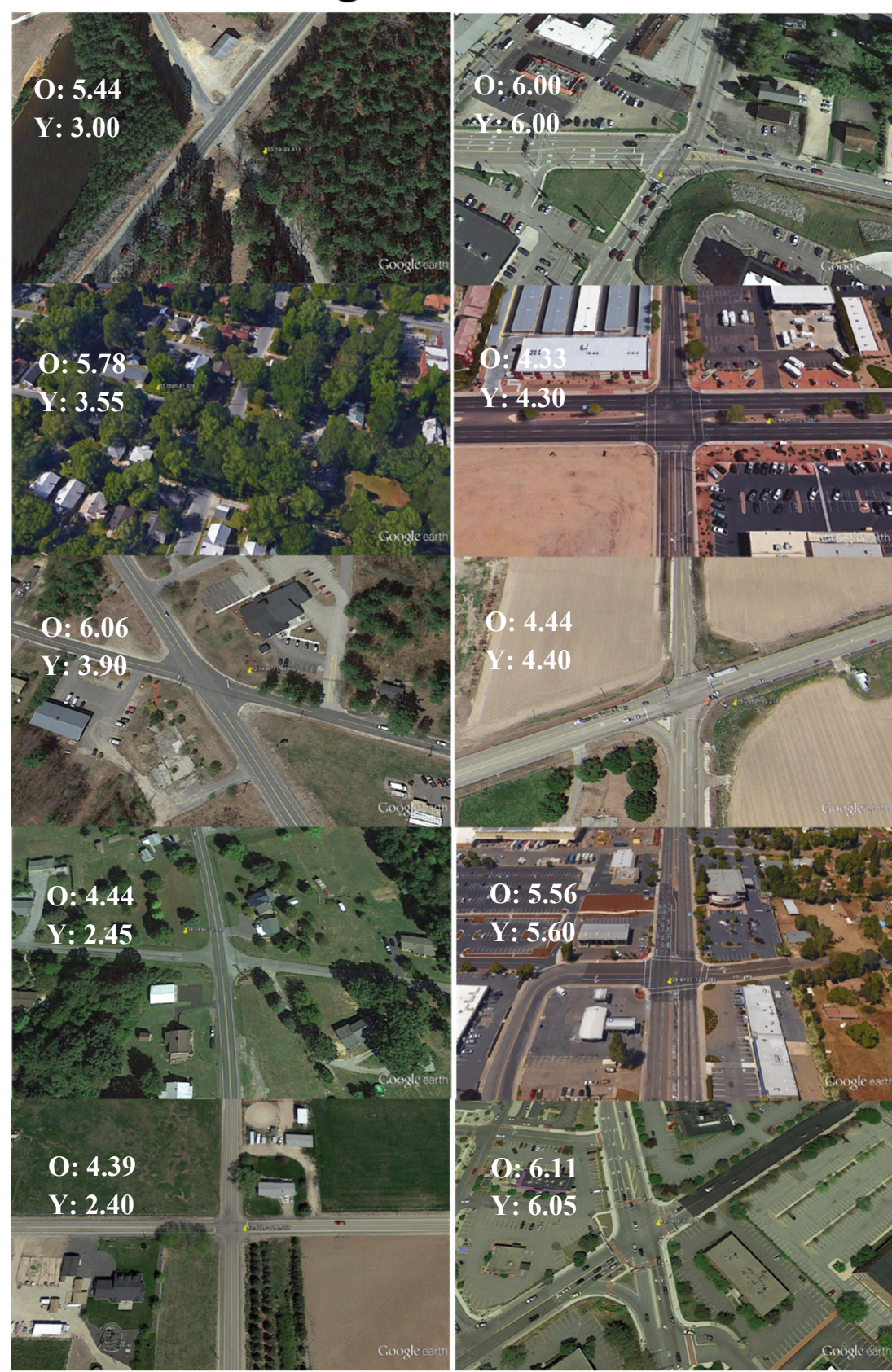

Younger $>$ Older

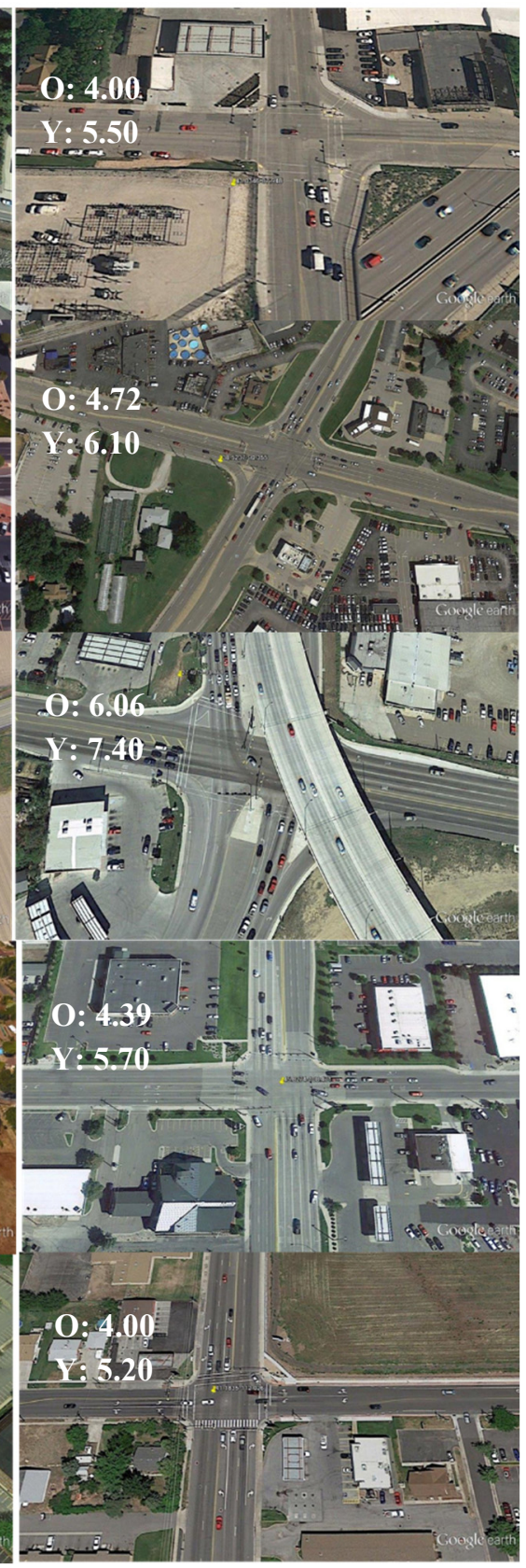

Figure 1. Age differences in intersection complexity ratings: the top five intersections representing the largest age differences with older adults rating the intersections as more complex (left column), the five intersections representing the smallest age differences (middle column), and the top five intersections with the largest age differences with younger adults rating the intersections as more complex (right column). The mean complexity ratings of each image are in the upper left corner of the respective image for both older $(O)$ and younger (Y) drivers 
consistent with findings suggesting older adults utilize distinct strategies from younger adults in the driving context (Wright et al., 2015), and more specifically, is consistent with prior work showing experienced drivers are more likely to anticipate hazards than younger drivers (Pradhan et al., 2005; Wright et al., 2016). The hazards that older drivers differentially anticipated are often less obvious in rural or residential streets.

One strength of the current approach is to generate lab-based stimuli based on real-world crash locations from FARS. The study described here represents an initial application and preliminary analysis. Future iterations will stratify the image sets based on the age of crash-involved drivers. For example, we will be able to oversample those intersection crashes where older or younger drivers were involved in order to examine the mapping to a variety of subjective ratings. This will allow for the confirmation of age differences in strategies or complexity rating criteria. Future iterations will also stratify the image sets based on objective parameters that are expected to influence complexity (e.g., traffic environment or number of lanes). This initial application took a somewhat arbitrary and circular approach in classifying intersection complexity, but this approach can be refined by the above stratification or through the use of an expert panel to define intersection complexity a priori (this was done, in part, here). Once an objective criterion for intersection complexity is defined, instead of the simple averaging of a quantitative rating, age differences can be examined at both the high end and low end of the scale. Concurrent or retrospective verbal reports would also likely be beneficial in elucidating the hypothesized differences, but due to time constraints, were not included in the current study. Finally, the overhead images in the current study were selected due to limitations of Google Earth, and it is unclear the extent the current study's results provide insights about intersection complexity from the driver's perspective. The current study was designed to address this limitation and additional analyses of both the overhead and the driver-perspective rating data will provide insight to the extent these preliminary findings replicate with more ecologically valid scenes.

\section{ACKNOWLEDGMENTS}

The authors would like to acknowledge Andrew Chang for his assistance in stimuli preparation, data collection, and data processing and to Marvin Dainoff for his comments and suggestions.

\section{REFERENCES}

Fleiss, J. L. (1981). Statistical methods for rates and proportions. 2nd ed. New York: John Wiley \& Sons, Inc.

Fuller, R. (2005). Towards a general theory of driver behaviour. Accident Analysis \& Prevention, 37(3), 461-472.Li, G., Braver, E.R., \& Chen, L. (2003). Fragility versus excessive crash involvement as determinants of high death rates per vehicle-mile of travel among older drivers. Accident Analysis \& Prevention, 35, 227-235.

Li, G., Braver, E.R., \& Chen, L. (2003). Fragility versus excessive crash involvement as determinants of high death rates per vehicle-mile of travel among older drivers. Accident Analysis \& Prevention, 35, 227-235.

Lombardi, D. A., Horrey, W. J., \& Courtney, T. K. (2017). Age-related differences in fatal intersection crashes in the United States. Accident Analysis \& Prevention, 99, 20-29. 
Owsley, C., Ball, K., McGwin Jr, G., Sloane, M. E., Roenker, D. L., White, M. F., \& Overley, E. T. (1998). Visual processing impairment and risk of motor vehicle crash among older adults. Jama, 279(14), 1083-1088.

Pradhan, A. K., Hammel, K. R., DeRamus, R., Pollatsek, A., Noyce, D. A., \& Fisher, D. L. (2005). Using eye movements to evaluate effects of driver age on risk perception in a driving simulator. Human Factors: The Journal of the Human Factors and Ergonomics Society, 47(4), 840-852.

Salthouse, T. A. (2004). What and when of cognitive aging. Current Directions in Psychological Science, 13(4), 140-144.

Wright, T. J., Samuel, S., Borowsky, A., Zilberstein, S., \& Fisher, D. L. (2016, September). Experienced drivers are quicker to achieve situation awareness than inexperienced drivers in situations of transfer of control within a Level 3 autonomous environment. In Proceedings of the Human Factors and Ergonomics Society Annual Meeting (Vol. 60, No. 1, pp. 270-273). SAGE Publications.

Wright, T. J., Vitale, T., Boot, W. R., \& Charness, N. (2015). The impact of red light running camera flashes on younger and older drivers' attention and oculomotor control. Psychology and Aging, 30(4), 755. 